Государственный институт усовершенствования врачей МО РФ, Москва

\title{
A.I.Sinopalnikov, I.L.Klyachkina, M.B.Mironov \\ Beclason ECO Easy Breathe: new possibilities of known drug in treatment of asthma
}

\section{Summary}

An open comparative trial was designed to compare clinical efficacy of beclomethasone dipropionate (BDP) via non-freon metered dose inhaler (MDI) Easy Breathe or freon-containing MDI.

The trial involved 30 patients not younger than 18 yrs with stable moderate to severe bronchial asthma (BA). The length of the disease exceeded 12 months and duration of previous therapy with inhaled steroids (freon-containing BDP) 1000 to 1500 mcg daily was at least 4 months. The trial duration was 6 months. The patients were randomised into 2 groups, 15 patients in each. Clinical signs, peak expiratory flow rate, need in short-acting $\beta_{2}$-agonists were monitored. The study group patients were given non-freon BDP (Easy Breathe) instead of freon-containing BDP in the ratio $1: 1$. The control group patients continued treatment with freon-containing BDP. Then BDP daily doses were gradually reduced in both the groups while BA was controlled adequately. The daily dose was reduced by $500 \mathrm{mcg}$ in average in 11 of $15(73.3 \%)$ non-freon BDP patients and in $6(40 \%)$ freon-containing BPD patients.

So, BDP via MDI ECO Easy Breathe allows moderate to severe BA to be controlled with lower doses of the drug. This reduces a cost of the therapy, rate of potential adverse effects and results in improvement of quality of life of the patients.

\section{Резюме}

Нами было проведено открытое сравнительное исследование, цель которого состояла в сопоставлении клинической эффективности беклометазона дипропионата (БДП), доставляемого дозированным аэрозольным ингалятором (ДАИ) «Легкое дыхание» на бесфреоновой основе (ГФУ) и фреонсодержащими (ХФУ) ДАИ.

В исследовании приняли участие 30 больных не моложе 18 лет с бронхиальной астмой (БА) среднетяжелого и тяжелого течения в стабильном состоянии. Длительность заболевания во всех случаях превышала 12 мес., а предшествующая терапия ингаляцинными глюкокортикостероидами (иГКС) в форме БДП-ХФУ в дозах от 1000 до 1500 мкг / сут. составляла не менее 4 мес. Длительность исследования составила 6 мес.

Больные были рандомизированы в 2 группы по 15 человек. Контроль состояния осушествлялся по клиническому статусу, пикфлоуметрии, потребности в $\beta_{2}$-агонистах короткого действия. У пациентов основной группы БДП-ХФУ был заменен на БДПГФУ в форме ДАИ «Легкое дыхание» в соотношении дозы $1: 1$; контрольная группа пациентов продолжала принимать БДПХФУ. В дальнейшем при адекватном контроле БА осуществлялось последовательное снижение суточной дозы БДП в обеих группах.

Суточная доза была снижена в среднем на 500 мкг у 11 из 15 (73,3\%) пациентов, получавших БДП-ГФУ, и только у 6 (40 \%) - в группе БДП-ХФУ.

Таким образом, применение БДП в виде ДАИ ЭКО «Легкое дыхание» дает возможность успешно контролировать БА среднетяжелого / тяжелого течения более низкими дозами иГКС, что уменьшает стоимость лечения, сокращает частоту возможных нежелательных явлений и, как следствие, улучшает качество жизни пациентов.

Бронхиальная астма (БА) - одно из наиболее распространенных хронических заболеваний как детей, так и взрослых, представляющее значительную медико-социальную проблему. Так, в частности, распространенность БА среди взрослого населения России достигает $5 \%[1,2]$.

Согласно современным представлениям, БА это хроническое воспалительное заболевание дыхательных путей, сопровождающееся развитием гиперреактивности бронхов, вследствие чего возникают повторяющиеся эпизоды свистящих хрипов, одышки, чувства стеснения в груди и кашля, особенно ночью или ранним утром. Эти эпизоды обычно связаны с распространенной, но изменяющейся по своей выраженности обратимой бронхиальной обструкцией [1].

В лечении больных БА ведущим является ин- галяционный способ лечения, преимущества которого заключаются в непосредственной доставке препарата к органу-мишени - слизистой дыхательных путей - и быстром начале действия лекарственного средства (в большинстве случаев через 5-20 мин). Доза лекарственного средства, вводимого ингаляционно, в 20 раз меньше дозы соответствующего препарата, принимаемого внутрь, что минимизирует вероятность развития нежелательных явлений. К тому же применение ингаляционного способа введения лекарственных средств улучшает качество жизни пациента.

Современный этап лечения БА берет свое начало с середины 1950-х гг., когда был создан первый дозированный аэрозольный ингалятор (ДАИ) «Medihaler» [3]. В качестве пропеллента для этого ингаляционного устройства был выбран фреон 


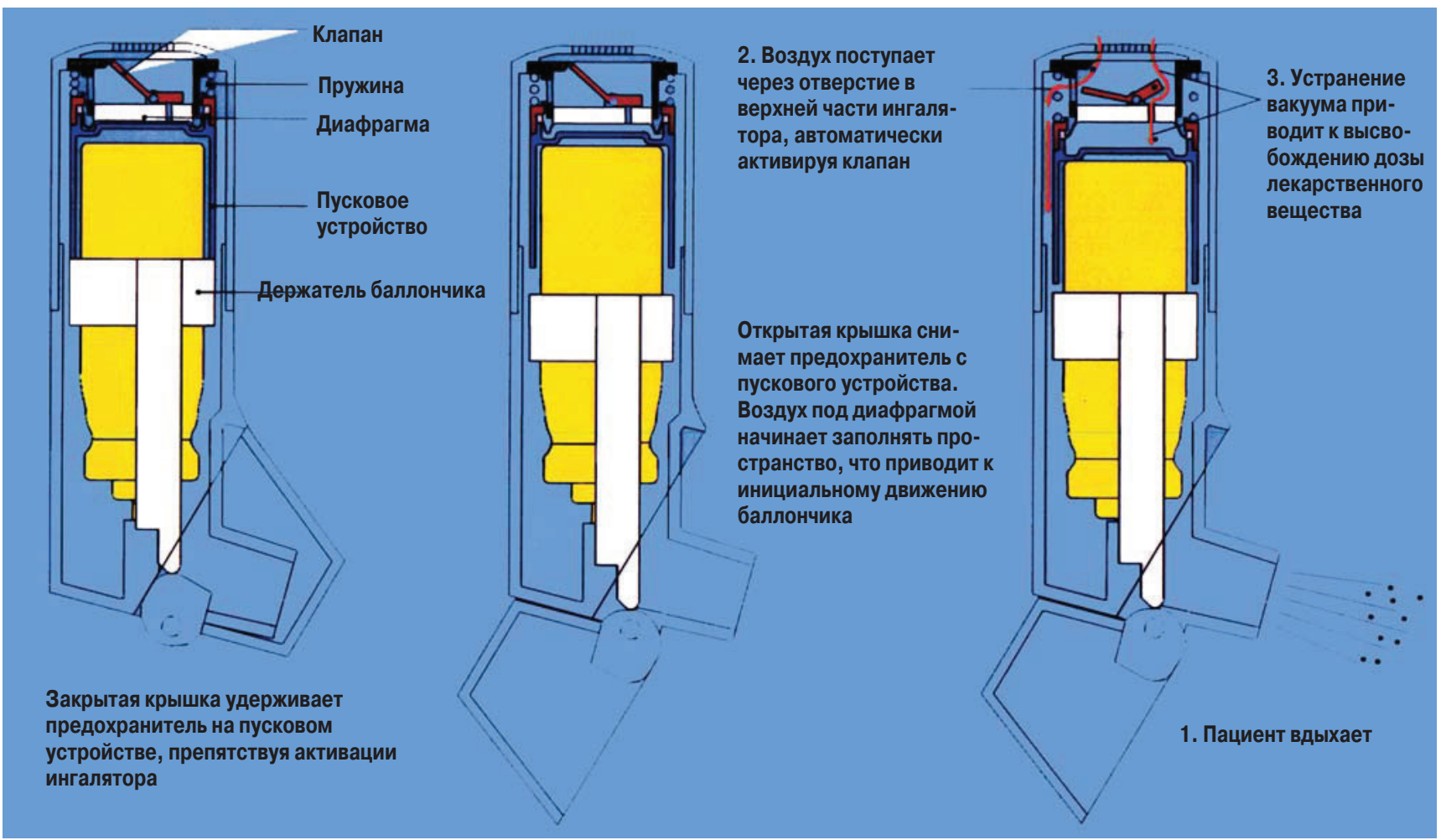

Рис. 1. Устройство ДАИ «Легкое дыхание»

(хлорфторуглерод - ХФУ). Простота устройства, отсутствие необходимости заряжать ингалятор, относительная дешевизна, портативность, гигиеничность быстро сделали ДАИ необходимыми и незаменимыми для больных БА. В настоящее время ежегодно выпускается более 500000 ДАИ; им отдают предпочтение более $70 \%$ больных БА во всем мире. Такому широкому распространению ДАИ не помешали даже их отдельные недостатки - прежде всего необходимость координации актуации ингалятора и медленного вдоха, что может привести к нарушению техники использования ДАИ и значительным потерям лекарственного средства $[4,5,6]$.

Впрочем, с появлением в 1970-х гг. дыхательных «помощников» - спейсеров и кондукторов отдельные недостатки ДАИ удалось преодолеть. Так, при использовании спейсера отпадает необходимость в координации вдоха и актуации ингалятора, несколько повышается температура аэрозоля и отсутствует быстрое поступление его частиц в ротоглотку. Дело в том, что на стенках спейсера оседают крупные частицы, уменьшается депозиция ингалируемого лекарственного средства на слизистой ротоглотки и, как следствие этого, минимизируется системная абсорбция. Комбинация ДАИ со спейсером большого объема (более 750 мл) дает возможность ингалировать большие дозы бронхолитиков, что особенно актуально при купировании тяжелых приступов БА. Эффективность этой комбинации сопоставима с таковой при использовании небулайзеров [7].

Применение спейсера уменьшает также депо- зицию преимущественно крупных частиц глюкокортикостероидов в ротоглотке (до $80 \%$ дозы), что предупреждает развитие таких нежелательных явлений, как кандидоз слизистой полости рта, дисфония, и способствует увеличению респирабельной фракции препарата.

В последние годы для изготовления ДАИ используются другие пропелленты - гидрофторуглероды (ГФУ), что продиктовано соответствующим положением Монреальского протокола «Субстанции, вызывающие истощение озонового слоя» (1989).

Для преодоления проблемы координации вдоха и активации ингалятора в 1960-е гг. были созданы дозированные пудросодержащие или порошковые ингаляторы (ДПИ), однако необходимость создания высокой объемной скорости инспираторного потока ограничивает возможность применения этих ингаляторов у больных с тяжелым течением БА [7, 8].

Как известно, больные БА развивают довольно низкую объемную скорость вдоха (ОСВ). Так, при оценке различных ДПИ (Турбухалер, Мультидиск) А.Г.Чучалин и соавт. [9] показали, что около $16 \%$ всех больных БА не в состоянии развить ОСВ > 60 л / мин (среди них - каждый второй больной с тяжелым течением заболевания). Примечательно, что при этом отсутствовала корреляция между показателями пиковой скоростью выдоха (ПСВ), измеряемой с помощью пикфлоуметра, и актуальными значениями значением OCB (In-chek inhaler assessment kit).

Наконец, в 1990 г. компанией «Norton» (впо- 
следствии - «IVAX») был разработан активируемый вдохом ДАИ, получивший название «EasyBreath» («Легкое дыхание»). Особенностью этого ингалятора является наличие пружинного механизма, который взводится открытием колпачка (рис. 1). После вдоха в течение 0,2 с происходит высвобождение фиксированной дозы препарата. Для активации ингалятора пациенту достаточно развить ОСВ порядка 10-25 л / мин. Эта характеристика устройства делает его доступным для большинства больных БА, даже с тяжелом течением заболевания [10].

Ингалятор ЭКО «Легкое дыхание» поставляется в комплекте с небольшим спейсером Optimizer (объем 50 мл). Использование последнего имеет большое значение для уменьшения орофарингеальной депозиции препаратов и, как следствие этого, минимизации частоты системных эффектов при применении, в частности, ингаляционных глюкокортикостероидов (иГКС) $[9,11]$.

Респирабельная фракция лекарственных средств, применяемых в ДАИ ЭКО «Легкое дыхание», вдвое превышает таковую при использовании обычных ДАИ и составляет $18-21 \%$ от ингалируемой дозы [12]. Важно при этом подчеркнуть, что респирабельная фракция не зависит от усилий пациента и актуальных значений ОСВ, в то время как при использовании ДПИ разброс ее значений колеблется от 9 до $28 \%$ и в значительной степени зависит от усилий больного [13]. В то же время использование спейсера не приводит к уменьшению легочной (респирабельной) фракции препарата [11].

Клинические исследования показали, что больные очень быстро обучаются технике ингаляционного маневра при использовании ДАИ, активируемых вдохом [14]. Так, в частности, J.Lenney et al. [15] показали хорошую технику ингаляции с помощью ДАИ ЭКО «Легкое дыхание» у $91 \%$ больных БА.

Исходя из концепции о воспалительном генезе БА, ведущим направлением базисной терапии заболевания являются иГКС (беклометазон пропионат, будесонид, флютиказон пропионат и др.) [1]. Среди них следует отметить беклометазон дипропионат (БДП), первый из иГКС, созданный в начале 1970-х гг. и до настоящего времени остающийся «золотым стандартом» противоастматической терапии [16].

Говоря о ДАИ, сегодня нельзя не отметить тот факт, что бесфреоновые аэрозольгенераторы способны улучшить эффективность уже известных субстанций, что объясняется увеличением дисперсности аэрозоля и респирабельной фракции ингалируемых лекарственных средств [17].

Так, в частности, при сравнении «низкодозного» (прием - 400 мкг БДП-ГФУ или БДП-ХФУ в день в течение 6 нед.) и «высокодозного» (прием 1200 мкг БДП-ГФУ vs. БДП-ХФУ в день в тече- ние 12 нед.) лечебных режимов оказалось, что они сопоставимы по влиянию на регресс клинических симптомов, улучшение показателей функции внешнего дыхания - ФВД - (в частности, объема форсированного выдоха за 1-ю с - OФВ 1 , и пиковой скорости выдоха - ПСВ), уровень кортизола в крови, частоту нежелательных явлений, в т. ч. и колонизацию ротоглотки Candida spp. [18].

Сходные результаты были получены и при изучении сравнительной эффективности БДП-ХФУ и БДП-ГФУ с помощью ДАИ «Легкое дыхание» у детей, страдающих БА [19].

Эти и ряд других исследований показали, что переход с БДП-ХФУ на БДП-ГФУ возможен в эквивалентных дозах $(1: 1)$ без снижения эффективности контроля над симптомами БА. Увеличение ингалируемого лекарственного средства при переходе на бесфреоновый ДАИ респирабельной фракции дало надежду на возможное снижение дозы иГКС, необходимой для контроля над симптомами БА.

Для подтверждения этой гипотезы было проведено рандомизированное исследование в параллельных группах больных БА с назначением БДП-ХФУ и БДП-ГФУ в дозах по 100, 400 и 800 мкг / день. При оценке динамики клинических симптомов, параметров ФВД, количества ингаляций $\beta_{2}$-агонистов «по требованию» и других было установлено, что адекватный контроль над течением заболевания достигается при дозах БДП-ГФУ меньших чем БДП-ХФУ в 2,6 раза [20]. Полученные данные позволили авторам высказать предположение о возможности перехода с фреонового на бесфреоновый ДАИ в соотношении доз иГКС $-2: 1$.

Для проверки этого предположения нами было проведено открытое сравнительное исследование, цель которого состояла в сопоставлении клинической эффективности БДП, доставляемым ДАИ «Легкое дыхание» на основе ГФУ и фреонсодержащими ДАИ.

В исследовании, проходившем в период с сентября 2003 г. по июнь 2004 г., приняли участие 30 больных БА среднетяжелого и тяжелого течения в возрасте $>18$ лет. Длительность заболевания во всех случаях превышала 12 мес., а предшествующая терапия иГКС в форме БДП-ХФУ в дозах от 1000 до 1500 мкг / день составляла не менее 4 мес. Важно подчеркнуть, что к моменту включения в исследование у всех пациентов на протяжении последних 3 мес. сохранялось контролируемое течение БА: отсутствовали обострения заболевания, требовавшие приема ГКС внутрь, незапланированные посещения врача, госпитализации, пробуждения вследствие развития эпизодов респираторного дискомфорта, а ежесуточное использование $\beta_{2}$-агонистов короткого действия по требованию не превышало 4 ингаляций. Кроме этого, все пациенты при использовании ДАИ демонстрировали правильную технику 


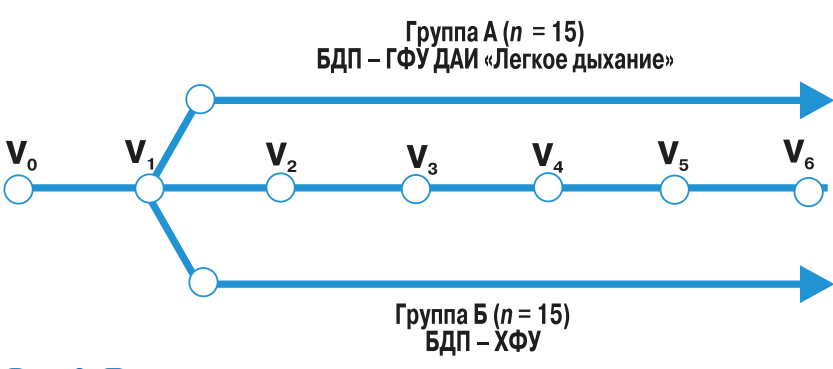

Рис. 2. Дизайн исследования

ингаляционного маневра и адекватно оценивали свое состояние.

Дизайн исследования предполагал 6 последовательных визитов больного (рис. 2). Включению пациентов в исследование предшествовал 10 -дневный вводный период $\left(\mathrm{V}_{0}-\mathrm{V}_{1}\right)$, в ходе которого подтверждалось стабильное течение заболевания:

- суточная вариабельность ПСВ $<20 \%$;

- использование $\beta_{2}$-агонистов короткого действия по требованию $<4$ инг. / сут.;

- отсутствие пробуждений;

- отсутствие необходимости в приеме системных ГКС;

- комплаентность иГКС (БДП) $>75 \%$, но $<125 \%$.

На 1-м визите $V_{1}$ больные были рандомизированы в 2 группы, состоявшие из 15 человек каждая (табл. 1). Пациенты в обеих группах на протяжении всего исследования (6 мес.) вели дневник самонаблюдения, в котором каждый день регистрировали динамику клинических симптомов, утренние и вечерние значения ПСВ, количество используемых по требованию $\beta_{2}$-агонистов короткого действия и др.

Во время исследования пациенты использовали индивидуальные пикфлоуметры («mini-Wright peak flow meter», «Clement Clarke Int. Ltd.», Англия). Ежедневно в утренние и вечерние часы (с 12-часовым интервалом, примерно в одно и то же время) пациент выполнял 3 последовательных измерения ПСВ и лучший результат заносил в дневник самонаблюдения.

У пациентов основной группы (группа A) на 1-м визите $\left(\mathrm{V}_{1}\right)$ была проведена смена терапии с отменой БДП-ХФУ и назначением БДП-ГФУ (в форме ДАИ «Легкое дыхание») в соотношении дозы $1: 1$; контрольная группа пациентов (группа Б) продолжала принимать БДП-ХФУ.

Врачебный контроль осуществлялся на каждом из последующих 5 визитов $\left(\mathrm{V}_{2}-\mathrm{V}_{6}\right)$, во время которых наряду с опросом, осмотром больного, изучением записей в дневнике самонаблюдения исследовалась ФВД (спирометр «SHILLER SR1», «Shiller $A G »$, Швейцария).

На 3-м визите $\left(\mathrm{V}_{3}\right)$ (т. е. через 3 мес. от начала исследования) у пациентов с адекватно контролируемой БА осуществляли редукцию суточной дозы БДП - на 250 мкг - для пациентов обеих
Таблица 1

Характеристика больных БА основной и контрольной групп

\begin{tabular}{|l|l|c|c|}
\hline \multicolumn{2}{|c|}{ Учитываемый признак } & $\begin{array}{c}\text { Основная } \\
\text { группа (А) }\end{array}$ & $\begin{array}{c}\text { Контрольная } \\
\text { группа (Б) }\end{array}$ \\
\hline \multicolumn{2}{|l|}{ Количество больных } & $15 / 100 \%$ & $15 / 100 \%$ \\
\hline \multicolumn{2}{|c|}{ Средний возраст (лет) } & $66,0 \pm 7,0$ & $64,0 \pm 14,0$ \\
\hline \multirow{2}{*}{ Пол } & женский & $5 / 33,3 \%$ & $5 / 33,3 \%$ \\
\cline { 2 - 4 } & мужской & $10 / 66,7 \%$ & $10 / 66,7 \%$ \\
\hline \multirow{2}{*}{ Форма БА } & $\begin{array}{l}\text { неустановлен- } \\
\text { ной этиологии }\end{array}$ & $14 / 93,3 \%$ & $14 / 93,3 \%$ \\
\cline { 2 - 4 } & атопическая & $1 / 6,7 \%$ & $1 / 6,7 \%$ \\
\hline $\begin{array}{l}\text { Степень тяже- } \\
\text { сти заболе- } \\
\text { вания }\end{array}$ & средняя & $10 / 66,7 \%$ & $12 / 80 \%$ \\
\cline { 2 - 4 } & тяжелая & $5 / 33,3 \%$ & $3 / 20 \%$ \\
\hline
\end{tabular}

групп. Таким образом, запланированная минимальная редукция дозы препарата для пациентов обеих групп составила:

- при первоначальной дозе 1000 мкг / сут. $25 \%$;

- при первоначальной дозе 1250 мкг / сут. $20 \%$;

- при первоначальной дозе 1500 мкг / сут. $17 \%$.

В случае возникновения нетяжелого обострения БА ${ }^{1}$, которое само по себе не служило причиной прекращения участия пациента в исследовании, он возвращался к первоначальной дозировке иГКС.

На последующих визитах $\left(\mathrm{V}_{4}, \mathrm{~V}_{5}, \mathrm{~V}_{6}\right)$ при отсутствии признаков обострения БА нами проводилась последовательная редукция суточной дозы БДП - всякий раз на 250 мкг. При возникновении нетяжелого обострения БА мы возвращались к дозировке иГКС, имевшей место на предыдущем визите. При стабилизации состояния и самочувствия пациента на следующем визите вновь предпринималась попытка редукции дозы препарата.

Статистический анализ полученных результатов предполагал использование следующих методов: $\chi^{2}$-критерий Пирсона, t-критерий Стюдента, критерий Шиффе - множественных сравнений. Все расчеты выполнены на персональном компьютере с использованием приложения Microsoft Excel и пакета статистического анализа данных Statistica 5.1 for Windows ( «Stat Soft Inc.», США).

Положительный результат определялся нами как достижение критериев контролируемой БА, а именно - отсутствие обострения заболевания, требуюшего приема ГКС внутрь, отсутствие незапланированных посещений врача, госпитализа-

${ }^{1}$ - Суточная вариабельность ПСВ > $20 \%$ и (или) использование $\beta_{2}$-агонистов короткого действия по требованию $>4$ раз / сут. и / или пробуждение в ночные или предутренние часы изза развития астматического приступа / эпизода респираторного дискомфорта, наблюдавшиеся в течение $\leq 2$ последовательных дней. 
таблииа 2

Распределение пащиентов БА в зависимости от привычной суточной дозы БДП на момент включения в исследование $\left(V_{p}\right)$

\begin{tabular}{|l|c|c|}
\hline Группы пациентов & Доза БДП, мкг / сут. & $n / \%$ \\
\hline \multirow{3}{*}{ Основная (А) } & 1000 & $6 / 40,0$ \\
\cline { 2 - 3 } & 1250 & $5 / 33,3$ \\
\hline \multirow{3}{*}{ Контрольная (Б) } & 1500 & $4 / 26,7$ \\
\cline { 2 - 3 } & 1000 & $12 / 80,0$ \\
\cline { 2 - 3 } & 1250 & $2 / 13,3$ \\
\hline
\end{tabular}

\begin{tabular}{|l|c|c|}
\hline Группы пациентов & Доза БДП, мкг / сут. & $n / \%$ \\
\hline \multirow{3}{*}{ Основная (А) } & 500 & $5 / 33,3$ \\
\cline { 2 - 3 } & 750 & $6 / 40,0$ \\
\cline { 2 - 3 } & 1000 & $3 / 20,0$ \\
\cline { 2 - 3 } & 1250 & $1 / 6,7$ \\
\hline \multirow{3}{*}{ Контрольная (Б) } & 500 & $4 / 26,7$ \\
\cline { 2 - 3 } & 750 & $7 / 46,7$ \\
\cline { 2 - 3 } & 1000 & $4 / 26,7$ \\
\cline { 2 - 3 } & 1250 & $0 / 0$ \\
\hline
\end{tabular}

Таблица 4

Распределение пациентов в зависимости от степени редукции суточной дозы БДП в процессе исследования $\left(V_{3}-V_{6}\right)$

\begin{tabular}{|l|c|c|c|c|}
\hline \multirow{2}{*}{ Группы пациентов } & \multicolumn{4}{|c|}{ Снижение суточной дозы БдП на } \\
\cline { 2 - 5 } & 0 мкг & 250 мкг & 500 мкг & 750 мкг \\
\hline Основная (А) & $0 / 0 \%$ & $3 / 20 \%$ & $11 / 73,3 \%{ }^{*}$ & $1 / 6,7 \%$ \\
\hline Контрольная (Б) & $2 / 13,3 \%$ & $7 / 47 \%$ & $6 / 40 \% *$ & $0 / 0 \%$ \\
\hline
\end{tabular}

Примечание: * - межгрупповые различия статистически достоверны $(P=0,04)$.

ций, пробуждений, не более 2 ингаляций $\beta_{2}$-агониста короткого действия по требованию.

Под отрицательным результатом подразумевалось тяжелое обострение БА, предполагающее назначение ГКС внутрь и исключавшее дальнейшее участие пациента в исследовании. Необходимо отметить, что имевшие место случаи обострения заболевания в период 6-месячного проспективного наблюдения были расценены как нетяжелые, и все пациенты завершили исследование.

Распределение пациентов основной и контрольной групп в зависимости от привычной дозы БДП на момент включения в исследование представлено в табл. 2.

Как уже говорилось выше, во время 3-го визита $\left(\mathrm{V}_{3}\right)$ предпринималась 1-я попытка редукция дозы иГКС на 250 мкг / сут. для пациентов обеих групп. Минимальную редукцию суточной дозы БДП - на 250 мкг - удалось осуществить у всех пациентов основной группы и у 13 из 15 (86,7 \%) пациентов контрольной группы. Попытки редукции суточной дозы БДП предпринимались и на последующих визитах $\left(\mathrm{V}_{4}, \mathrm{~V}_{5}\right.$ и $\left.\mathrm{V}_{6}\right)$. Как показал анализ полученных данных, достоверных различий в распределении пациентов основной и контрольной групп в зависимости от суточной дозы БДП, контролировавшей течение БА на последнем визите $\left(\mathrm{V}_{6}\right)$, выявлено не было (табл. 3).

Вместе с тем, анализ распределения пациентов сравниваемых групп в зависимости от степени достигнутой редукции суточной дозы БДП свидетельствовал, что наибольшее снижение (на 500 мкг) имело место при переходе пациентов на БДП-ГФУ (ДАИ «Легкое дыхание») - табл. 4.
Таблица 5

Среднесуточная доза БДП у пациентов БА основной и контрольной групп (в целом по группе) в начале $\left(V_{1}\right)$ и в конще (V) исследования

\begin{tabular}{|l|c|c|c|}
\hline \multirow{2}{*}{ Группы пациентов } & \multicolumn{3}{|c|}{ Среднесуточная доза БДП, мкг } \\
\cline { 2 - 4 } & $\mathbf{V}_{1}$ & $\mathbf{V}_{6}$ & $\mathbf{V}_{1}-\mathbf{V}_{6}$ \\
\hline Основная группа & $1217 \pm 208 *$ & $750 \pm 231$ & $467 \pm 153^{*}$ \\
\hline Контрольная группа & $1067 \pm 148$ * & $750 \pm 189$ & $317 \pm 127^{*}$ \\
\hline
\end{tabular}

Примечание: * - межгрупповые различия статистически достоверны $(\mathrm{P}<0,05)$.

Столь же демонстративными представлялись и различия среднесуточных доз БДП у пациентов основной и контрольной групп в начале и в конце исследования (табл. 5).

В целом прием больными БА среднетяжелого и тяжелого течения иГКС в течение 6 мес. в виде БДП (БДП-ГФУ ДАИ «Легкое дыхание» и БДП-ХФУ ДАИ) характеризовался хорошей переносимостью. Только у 2 пациентов (по 1 - из основной и контрольной групп) при приеме БДП в дозе 1000 мкг / сут. была отмечена осиплость голоса, и еще у 1 пациента контрольной группы развилась интеркуррентная респираторная вирусная инфекция.

Итак, согласно результатам проведенного исследования при назначении пациентам со стабильным течением среднетяжелой / тяжелой БА беклометазона дипропионата в виде ДАИ ЭКО «Легкое дыхание» (БДП-ГФУ) удалось снизить суточную дозу иГКС у большинства пациентов у 11 из 15 (73,3\%) - в среднем на 500 мкг. Для сравнения, подобная степень редукции суточной дозы иГКС в группе пациентов БА, получавших 
БДП-ХФУ, была достигнута только в 6 случаях $(40,0 \%)$.

Таким образом, применение БДП в виде ДАИ ЭКО «Легкое дыхание» дает возможность успешно контролировать течение БА среднетяжелого / тяжелого течения более низкими дозами иГКС, что будет способствовать уменьшению стоимости лечения, сокращению частоты возможных нежелательных явлений и, как следствие этого, улучшению качества жизни пациентов.

\section{Литература}

1. Бронхиальная астма. Глобальная стратегия. Национальный Институт Сердце, Легкие и Кровь. Издание № 95-3659. Январь 1995. Русская версия под ред. акад. А.Г.Чучалина. Пульмонология. 1996.

2. Чучалин А.Г., Медников Б.Л., Белевский А.С. и др. Бронхиальная астма. Руководство для врачей России. (Формулярная система). Пульмонология 1999; Прил.

3. Huchon G. Metered dose inhalers: past and present: advantages and limitations. Eur. Respir. Rev. 1997; 41: $26-28$.

4. Chinet T., Huchon G. La mauvaise utilisation des aerosols-doseurs pressurises dans maladies bronchiques: frequence et consequence cliniques. Ann. Med. Int. 1994; 145: 119-124.

5. Goodman D.E., Israel E., Rosenberg M. et al. The influence of age, diagnosis and gender on proper use of metered-dose inhalers. Am. J. Respir. Care. Med. 1994; 150: 1256-1261.

6. Thompson J., Irvine T., Grathwohl K. et al. Misuse of metered-dose inhalers. Chest 1994; 105: 715-717.

7. Engel T., Heining J.H., Malling H.J. et al. Clinical comparison of inhaled budesonide delivered via pressurized metered-dose inhaler or Turbuhaler. Allergy 1989; 44: $220-225$.

8. Огородова Л.М. Системы ингаляционной доставки препаратов в дыхательные пути. Пульмонология 1999; 1: 84-87.

9. Chuchalin A.G., Sakharova G.M. et al. Eur. Respir. J. 2000; 16 (suppl. 31): 316S.
10. Crompton G.K., Duncan J. Clinical assessment of a new breath-actuated inhaler. Practitioner 1989; 233: 268-269.

11. Anhoj J., Bisgaard H., Lipwort B.J. Effect of electrostatic charge in plastic spacers on the lung delivery of HFA-salbutamol in children. Br. J. Clin. Pharmacol. 1999; 47: 333-336.

12. Hardy J.G., Jasuja A.K., Trier M. et al. A small volume spacer for the use with a breath - operated pressurised metered dose inhaler. Int. J. Pharmaceut. 1996; 142: 129-133.

13. Newman S.P., Weisz A.W.B., Talace N. et al. Improvement of drug delivery with a breath actuated pressured aerosol for patients with poor inhaler technique. Thorax 1991; 46: 712-716.

14. Cochrane M.J., Mohan M.D., Bala V. et al. Inhaled corticosteroides for asthma therapy patient compliance, devices, and inhalation technique. Chest 2000; 117 (2): $542-550$.

15. Lenney J., Innes J.A., Crompton G.K. Inappropriate inhaler use: assessment of use and patient preference of seven inhalation devices. EDICI. Respir. Med. 2000; 94: 496-500.

16. Brown H.V., Storey G., George W.H.S. Beclomethasone dipropionat: a new steroid aerosol for the treatment of allergic asthma. Br. Med. J. 1972; 1: 585-590.

17. Leach C.L. Improved thievery of inhaled steroids to the large and small airways. Respir. Med. 1998; 92 (suppl. A): $3-8$.

18. Milanovski J., Qualthrough J., Perrin V.L. Inhaled beclometasone (BDP) with non-CFC propellant for the treatment of astma. Respir. Med. 1999; 93: 245-251.

19. Farmer L.S., Middle M., Savic J. et al. Therapeutic equivalence of inhaled beclometasone dipropionate with CFC and non- CFC (HFA 134a) propellants both delivered via the «Easybreathe» TM inhaler for the treatment of pediatric asthma. Respir. Med. 2000; 94: 57-63.

20. Busse W.W., Brazinsky S., Jacobson K. et al. Efficacy response of inhaled beclometasone dipropionate in asthma is proportional to dose and is improved by formulation with a new propellant. J. Allergy Clin. Immunol. 1999; 104 (6): 1215-1222.

Поступила 30.12.04 () Коллектив авторов, 2005 удК 616.248-085.234 